\title{
Object-centred spatial reference in 4-month-old infants
}

\author{
Andrew J. Bremner a,b,., Peter E. Bryant c, Denis Mareschal b \\ a Department of Psychology, Whitehead Building, Goldsmiths, University of London, New Cross, \\ London SE14 6NW, UK b Centre for Brain and Cognitive Development, Birkbeck, University of \\ London, UK c Department of Experimental Psychology, University of Oxford, UK \\ Corresponding author at: Department of Psychology, Whitehead Building, Goldsmiths, \\ University of London, New Cross, London SE14 6NW, UK. Tel.: +44 207078 5074; fax: +44 207 \\ 919 7873. E-mail address: a.bremner@gold.ac.uk (A.J. Bremner).
}

\begin{abstract}
An appreciation of object-centred spatial relations involves representing a 'within-object' spatial relation across changes in the object orientation. This representational ability is important in adult object recognition [Biederman, I. (1987). Recognition-bycomponents: A theory of human image understanding. Psychological Review, 94, 115-147; Marr, D., \& Nishihara, H. K. (1978). Representation and recognition of the spatial organisation of three-dimensional structure. Proceedings of the Royal Society of London, Series B (Biological Sciences), 200, 269-294; Tarr, M. J., \& Pinker, S. (1990). When does human object recognition use a viewer-centred reference frame? Psychological Science, 1, 253-256] and is also thought to be a fundamental component of the mature object concept [Piaget, J. (1954). The Construction of Reality in the Child. Routledge \& Kegan-Paul: London, UK. (Originally published in French in 1937)]. An experiment is reported in which eighteen 4-month-old infants were familiarised to a specific spatial relation within an object, across six different orientations of the object. On subsequent test trials the object was presented to the infants in an entirely novel orientation. Between successive test trials the within-object spatial relation was alternated between novel and familiar. The infants demonstrated significant sensitivity of their looking to both the novelty of the stimuli and the order in which novel and familiar stimuli were presented. It is concluded that by 4 months of age infants are able to form object-centred spatial frames of reference. These findings are discussed in the light of our current understanding of the development of object representation during infancy.
\end{abstract}

Keywords: Object concept; Spatial representation; Infancy; Cognitive development; Object recognition 
The nature of our internal representations of 'object-centred' spatial coordinates is a topic of controversy and continued debate (Biederman, 1987; Mozer, 2002; Tarr, Williams, Hayward, \& Gautier, 1998; Tipper \& Behrmann, 1996; Vecera, Behrmann, \& Filapek, 2001). One view is that objects are encoded and stored relative to egocentric spatial coordinates (Tarr, 1999), and that the information in such egocentric representations is rich enough to provide reliable object recognition across a variety of changes in orientation relative to the observer (Mozer, 2002). On the other hand, following Marr and Nishihara (1978); Biederman (1987) has argued that certain ' 3 D volumes' can be described by the visual system in a view-invariant code. Support for these 'structural description' theories has come from neuropsychological evidence of specific impairments in object-centred spatial representation (Tipper \& Behrmann, 1996). Despite strong objections to a pure view-invariant code for object recognition (Tarr, 1999; Tarr and Bulthoff, 1998) there is still clear agreement that at least some degree of object-centred spatial representation must exist (Mozer, 2002; Tarr and Pinker, 1990). Indeed, some have suggested that the mature visual system uses both view-specific and view-invariant representations of objects (Hummel, 2000).

It seems likely that theway in which object representation skills develop in infancy and childhood will have important implications for how object recognition is achieved in adulthood. So it is surprising that little developmental research has been done on object recognition. Nonetheless, it has been considered. Piaget (1937/1954) provides some striking examples of situations in which infants appear to be unable to use object-centred spatial reference. Here he describes how Laurent, at 7 months, has difficulty with his bottle at feeding time:

Obs. 78. At 0;7 (4) I present the bottle in a vertical position to Laurent ... he looks at it from bottom to top, sees the nipple and immediately brings it towards his mouth. He sucks. I take it from his hands and present it to him horizontally; Laurent easily turns the bottle in a quarter circle and puts the nipple in his mouth ... I present the bottle upside down, Laurent seeing only the bottom and no longer perceiving the nipple; he looks at it for one or two seconds, begins to howl without making any attempt at reversal ... Laurent looks, begins to suck the glass (the bottom), and howls again (Piaget, 1937/1954, p.127).

Piaget's point here is that Laurent cannot understand that the manual rotations of which he is capable will allow him to search for the reverse side of an object. Although Laurent can obviously recognise the bottle in all orientations (his frustration at not being able to feed himself, and the ineffectual attempts he makes to achieve this are indicators of his recognition), it is clear that the schema through which he recognises it does not also serve him as a detailed enough spatial representation for action. The impression that is taken from this example is that infants, even after 7 months of age, form relatively primitive representations of objects, to the extent where they do not represent their constant spatial shape.

From Piaget's (1936/1952, 1937/1954) developmental perspective, a view-independent structural representation of objects is an essential part of attaining a conception of objects as being constant entities that are independent of the self. Piaget argued that initially young infants are severely limited in this respect by their dependence on egocentric spatial reference, and that the development of more complex schemas for action in the external environment prompts them to construct representations of coordinates relative to external spatial frameworks. Research directed at determining the spatial frames of reference available in infancy (utilising visual anticipation and manual search measures) seems to support this position. Acredolo (1978); Bertenthal, Campos, and Barrett (1984); Bremner (1978); Bremner and Bryant (1977); Bremner and Bryant (2001); Butterworth, Jarrett, and Hicks (1982); Gilmore and Johnson (1997a, 1997b) have all presented evidence indicating that infants progress from the use of primitive retinocentric or egocentric spatial reference to more external frames of reference as they get older, or receive more locomotor experience. ${ }^{1}$ This supports Piaget's assertion that infants' early representations of objects are largely egocentric.

${ }^{1}$ Though see Hermer \& Spelke, 1994, Newcombe (2003), Newcombe and Huttenlocher, 2000 for alternative opinions on the development of spatial representation in infancy. 
On the other hand, subsequent research into infants' representations of objects (mostly utilising looking time measures) produced results that suggest a quite different conclusion. The fixed-trial familiarisation technique (Slater, 1995) has been particularly illuminating concerning infants' ability to represent the constant stimulus properties of objects across changes in the proximal stimulation that they offer. In this procedure infants are familiarised to several (often six) variants in the proximal stimulation offered by an object of a fixed distal size or shape, and are then tested for a preference between two objects, of novel and familiar distal sizes or shapes. Using this technique, Slater and colleagues have shown that newborns can discriminate between objects on the basis of their real shape (the shape of an object that remains constant despite changes in an object's slant in relation to the observer's retina) (Slater \& Morison, 1985) and size (Slater, Mattock, \& Brown, 1990).

It also turns out that infants' early representations of spatial relations may not be as egocentrically biased as Piaget suggested. Using looking behaviour in a habituation-novelty design, Kaufman and Needham (1999) tested whether young infants could represent environmental spatial layout independently of egocentric coordinates. They habituated a group of 6.5-month-olds to an object placed at a particular location on a table. Each habituation trial consisted of the infant being moved into one particular location relative to the table and the object. Following habituation, the infants were either moved into view at the opposite side of the table, or they were moved back to the side from which they had already observed the scene during habituation. In both these test conditions the object was then presented in either a novel location relative to the table (new egocentric location), or a familiar location with respect to the table (old egocentric location). The 6-month-olds continued to habituate if the object stayed in the same allocentric location whether or not they had been moved around the table. However, no matter where the infants were positioned, when the object moved to a new allocentric location they dishabituated significantly. Kaufman and Needham (1999) concluded that 6.5-month-old infants are able to code allocentric spatial location.

Evidence that infants of this young age are able to encode aspects of their visual environment independently of the egocentric spatial array does not answer the question whether they are also able to code the constant spatial layout of an object across changes in its orientation, despite Piaget's observation related above. However, the spatial representation of objects, can be considered a separate and even more complicated problem than spatial representation of the environment. Objects, and especially manipulable objects, as well as moving in depth frequently change in their orientation relative to the observer and environment. As such, they do not retain a fixed spatial relation to either egocentric or environmental frames of reference.

Thus, in order to represent the spatial relations within objects, infants need to utilise a spatial frame of reference that is independent of retinocentric, egocentric (body-centred), and allocentric (environmental) coordinates. The current experiment set out to determine whether young (4month-old) infants can represent the spatial information in a within-object (object-centred) frame of reference.We used the fixed-trial familiarisation method to determine whether infants can perceive and encode the spatial location of a feature within an object's frame of reference. Following familiarisation to a single object-centred location (OCL), we tested discrimination by examining whether infants show a post-familiarisation preference for a novel over a familiar object-centred location. In order to determine whether infants can represent object-centred location independently of egocentric and allocentric reference frames, we tested this discrimination of object-centred locations across changes in the object's orientation relative to egocentric and allocentric frameworks. 


\section{Method}

\subsection{Stimuli}

A schematic diagram of the object that we used to present object-centred locations is shown in four orientations in Fig. 1. It was shaped in the form of a capital ' $T$ ', and thus comprised three limbs; one perpendicularly oriented in relation to the other two. All three limbs of the object were the same appearance as each other (apart from their spatial relation to the other limbs) and the same length from the point of intersection. Each limb also contained a marked location that was occupied by a light that could be switched on or off. These object-centred locations are labelled in Fig. 1 as OCLs 1-3. When illuminated these OCLs were identical in appearance, making it possible for us to manipulate the spatial relation of a feature (light) to the object framework, simply by changing the OCL that was illuminated. We could change the object's orientation by rotating it within the picture plane around the point of rotation (located at the intersection of the two main axes). By rotating the object we were able to present any one object-centred location in several different egocentric/allocentric locations. As each of the OCLs was equidistant from the point of rotation, each also had the capacity to occupy the same distribution of locations in egocentric/allocentric space, and thus we could equate them on this basis.

\subsection{Design}

In order to avoid confounding object-centred with egocentric/allocentric frameworks, we familiarised infants to a single object-centred location presented in six different orientations of the object. The variation of the object's orientation across the familiarisation phase is a measure taken to desensitise the infants to the object's relation to egocentric spatial coordinates.

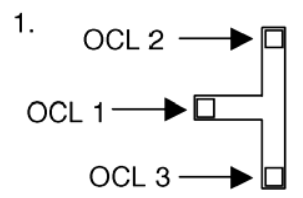

2.

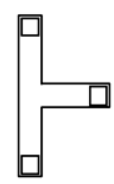

3.

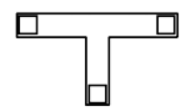

4.

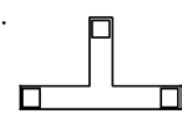

Fig. 1. Starting and test trial orientations of the T-shape, with object-centred locations (OCLs) labelled in orientation ' 1 '.

Familiarisation trials

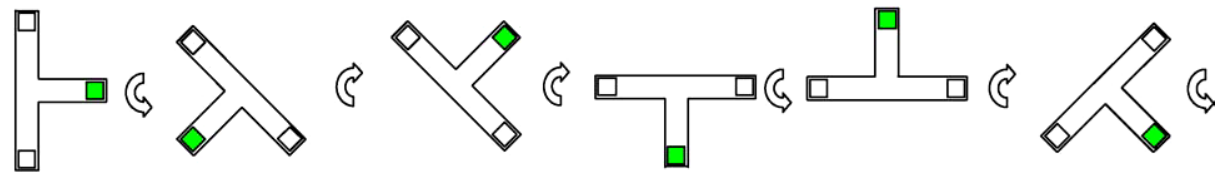

Test trials
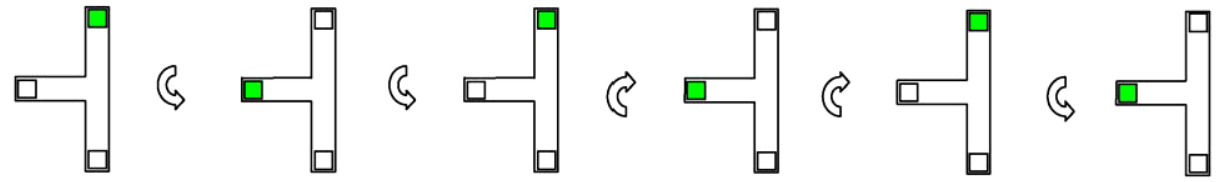

*Starting orientation 2, Order group 1, OCL comparison 1, and Novel OCL 2/3 (in this case '1')

Fig. 2. A schematic example of one experimental session (starting orientation 2, Order group 1, OCL comparison 1, and Novel OCL 2/3 (in this case ' 1 ')).

The familiarisation phase comprised six trials. The object was presented in a different orientation on each of these trials. For all participants the object underwent a fixed order of rotations between trials: Trials 1-2: 225. anticlockwise, trials 2-3: 180. clockwise, trials 3-4: 135. clockwise, trials 4-5: 180. anticlockwise, trials 5-6: 135. clockwise, trials 6-test: 225. anticlockwise. For each 
infant, the object started in one of four 'starting orientations' (see Fig. 1), and thus the orientation of the object on each trial depended on the starting orientation of the object.

On each familiarisation trial the same object-centred spatial location was lit up. Thus, the objectcentred location shown to each participant was invariant across all familiarisation trials. An example of familiarisation phase is shown in Fig. 2. In this example the familiarised objectcentred location is OCL 2 , and the starting orientation of the object is ' 2 '.

After familiarisation, the test phase presented novel and familiar OCLs within the T-object on successive trials. During the test phase we only drew novel-familiar contrasts between OCL 1 and OCL 2, or OCL 1 and OCL 3 (see Fig. 1). For successful discrimination, these particular contrasts require the representation of a spatial relation to only a single dimension or axis of the object. The object-centred contrast between OCLs 2 and 3 is only differentiated with respect to a coordinated representation of two of the object's axes. Tarr and Pinker (1990) have shown that adults recognise these more complex configural relations by using mental rotation to match against a stored egocentric representation. As we were testing object-centred spatial representation rather than egocentric spatial representation (with mental rotation) we excluded the $2 \mathrm{D}$ contrast from the current design. ${ }^{2}$

In order to be sure that a preference for the novel OCL could not be due to the novelty of the light in relation to the object in one particular orientation, we presented the object in a completely novel orientation in the test phase. We also wanted to be sure that a preference for the novel OCL could not be due to a novelty preference for the location of the light in relation to egocentric coordinates. Thus, at test we presented the object in such an orientation as to place the novel OCL in an egocentrically defined location that had already been occupied by the light during one of the familiarisation trials. The sequence of object orientations on familiarisation and test trials (see above) was arranged such that the familiar OCL was presented in an egocentric location that had not been occupied by the OCL light on any familiarisation trial.

The test phase also comprised six trials. For each participant the object was presented in the same orientation from trial to trial. We rotated the object through 36o. between each test trial. The directions of these rotations between each test trial were again in a fixed order for each participant: anticlockwise, anticlockwise, clockwise, clockwise, and anticlockwise.

The test phase was divided into three blocks of paired trials. In each block a novel object-centred location was highlighted on one trial, and the familiarised object-centred location (the OCL which a participant saw highlighted on all six familiarisation trials) on the other. Two order groups were presented with either the novel trial first within each block (order group 1), or the familiar trial first with each block (order group 2). Order group 1 was presented with the novel OCL on test trials 1, 3 and 5, and order group 2 with the novel OCL on test trials 2, 4 and 6.

Each participant saw the same novel OCL highlighted across all of the three novel trials. Half the infants was given a comparison between OCLs 1 and 2 (OCL comparison 1), and the other half between OCLs 1 and 3 (OCL comparison 2). This variable was called 'OCL Comparison'. The OCL which was assigned as the novel OCL in a given OCL comparison was also varied. Thus, infants were either familiarised to OCL 1 , or OCLs $2 / 3$, and were respectively assigned OCLs $2 / 3$, or OCL 1 as the novel OCL. This variable was called 'Novel OCL'.

The orders of the novel and familiar trials (x2), the OCL Comparison (x2), and Novel OCL Comparison (x2) were all counterbalanced across participants. The starting orientation (1-4) of the object was assigned to infants by a random allocation, with the restriction that there was an equal number of each of the starting orientations across the sample, and also within each order group.

2 The issue of mental rotation in infancy is addressed by Rochat and Hespos (1996) and Hespos and Rochat (1997). While their experiment also uses a 'T-shaped' stimulus they were not investigating spatial coding of a feature to an object-centred frame of reference, which is the question of interest here. 


\subsection{Participants}

Eighteen infants took part, of which 13 were female, and five male. Their mean age was 124 days (S.D. = 4.9). A further nine babies were tested but their data were excluded as five reached ceiling looking times on all test trials 3 , and four fussed too much for us to complete the test. Nine infants were tested in each order group. The mean age of the infants ( 7 female and 2 male) in order group 1 (novel OCL trial presented first) was 123 days (S.D. $=4.5)$. The mean age of the infants (6 female and 3 male) in order group 2 (novel OCL trial presented second) was 125 (S.D. $=5.4$ ). The infants who participated in this experiment lived were primarily from middle-class European-American families, and were selected on the basis of their parents volunteering to take part in the research programme.

\subsection{Apparatus}

All three limbs of the T-shaped object were $12.5 \mathrm{~cm}$ long from the point of intersection, $4 \mathrm{~cm}$ in width, and $4 \mathrm{~cm}$ in depth. Each OCL consisted of five green light emitting diodes (LEDs), fixed inside the T-shape and concealed (when not illuminated) behind a square window made from diffuse perspex. The object was mounted on a pole extending back in depth from the point of rotation. This pole was also mounted on a flat surface, so that the object's long axis moved within the frontal plane of an upright infant observer when it rotated. Everything behind the T-shape, including the pole, was concealed behind a black screen.

Behind the concealing screen the experimenter was able to change the orientation of the object unseen by rotating the pole. The experimenter was also able to observe the infant participants' visual aspect by way of a video camera mounted in the screen, about a foot above the object. Each infant viewed the object whilst sitting in an upright position on one of their parents' laps so that their eyes were roughly $60 \mathrm{~cm}$ from the stimulus. The seating position placed the infants with the object at their midlines. The longest axis of the T-shape presented roughly 22.6. of visual angle to the infant participants. Thus, if the infant were fixating the point of rotation, the end of each of the limbs extended roughly 11.3. into the periphery.

The lights in the T-shape were controlled automatically from a PC that also served as a millisecond timer for the infants' visual fixations. As the procedure involved infant-controlled familiarisation (i.e. familiarisation trials last until the infant has regarded the object for a fixed amount of time), we designed a programme which illuminated the stimulus lights and extinguished them automatically once the millisecond timer had registered a fixed amount of looking time.

\subsection{Procedure}

We thought it important to provide the infants with the same amount of exposure to each stimulus variant. Otherwise we might have run the risk of weighting their representations of object-centred location unevenly on one or two orientations of the object, and might consequently have overemphasised the inappropriate egocentric and allocentric frames of reference. Under some circumstances, the duration for which infants look at variants of stimuli has been found to decline over the familiarisation period (Bomba \& Siqueland, 1983; Quinn, Eimas, \& Tarr, 2002). Thus, in order to present the infants with equal exposure to the six variants, each trial was presented for a fixed amount of accumulated looking. This fixed-trial familiarisation procedure has been used in a similar form with newborns by Slater, Mattock, Brown, and Bremner (1991), and with newborns and 3-month-olds by Quinn, Slater, Brown, and Hayes (2001). Both groups found that six variants were sufficient to elicit a test trial novelty preference.

Before testing began, we asked the parent to try to keep the infant in a constant upright posture, and not to direct the child's attention during the procedure. We also asked them not to watch the stimulus presentation themselves, but instead to observe their child.

3 Infants who looked at the object for a total $80 \mathrm{~s}$ or more, out of a possible $90 \mathrm{~s}$ across the test phase were excluded. 
The experimental session began once parent and child were comfortably seated, and the child was settled. A curtain was lifted to reveal the object, and the first familiarisation trial began once the infant had fixated the object. The onset of each trial was signified by a short tone from the computer. Each familiarisation trial began with a single location lighting up (the familiarised OCL). When the infant had looked at the T-shape object (the whole object, not just the light) for $10 \mathrm{~s}$ (recorded by the experimenter with the millisecond timer linked to the control computer), the light was automatically turned off. This was also signalled by another short tone from the computer. The experimenter then rotated the T-shape to a new orientation (the rotation of the object was fully visible to the infant), and the next familiarisation trial would then begin (intertrial interval was set to $4 \mathrm{~s}$ ). For each infant, the light event appeared in the same object-centred location on every familiarisation trial.

Following six familiarisation trials, we presented the infants with six test trials. As in familiarisation the onset of each trial was signified by a tone from the computer, and the illumination of one OCL. However, unlike the familiarisation trials, each test trial lasted for $15 \mathrm{~s}$ of real time, during which the amount of time the infants spent looking at the whole object (not just the light) was recorded using the millisecond timer. The accuracy of test trial timings was later verified by referring to video recordings of the test trials.

When verifying infants' looking times from video recordings, the experimenter and the reliability observer were both blind to the familiarity/novelty of the test trials. Inter-observer reliabilitywas obtained by comparing the experimenter's and a reliability observer's total familiar and novel test-trial timings taken from the video records of 16 of the 18 infant test sessions. ${ }^{4}$ Inter-observer reliability ratings for novel and familiar test trials ranged between Pearson's $r=0.93$ and Pearson's $r=0.97$.

\section{Results}

Due to our fixed-trial accumulated looking procedure, all infants looked at the object for $10 \mathrm{~s}$ during each familiarisation trial. Thus, each looked at the object + light event for a total of $60 \mathrm{~s}$ during the familiarisation phase.

We calculated preference scores for each infant on each test trial block, by subtracting the amount of object-directed looking they demonstrated on the familiar trial from that they showed on the novel trial. Fig. 3 shows the infants' mean preference scores within each order group and test trial block. There is a positive preference score in 5 of the 6 test cells. However, the preference also appears to be dependent upon the order in which familiar and novel trials occur within each block. Infants in order group 1 demonstrate a consistent preference for the novel trial across all test blocks, whereas the infants in order group 2 appear to show a reduced or no novelty preference.

Because stimuli presented in the first test trials can affect novelty preferences in the later test trial blocks, we carried out an initial analysis of the looking times on only the values within the first block. These were entered into an ANOVA with 'order' as a between-subjects factor. Order did not reach significance $(F(1,16)=4.0, p>0.06)$. A two-tailed one-sample $t$-test revealed that the preference score was significantly greater than $\mathrm{O}$ on this trial $(t(17)=2.4, p=0.03)$.

Thus, on the first block, infants show a consistent novelty preference, indicating an ability to discriminate between familiar and novel object-centred spatial locations. The 18 infants who completed the test session presented data for a total of 54 novel-familiar test trial blocks.

\footnotetext{
4 The video records of two of the infants' test trial looking behaviour were lost before reliability was computed. However, as inter-observer reliability was high, we included the first observer's scores for these two infants in the analysis.
} 


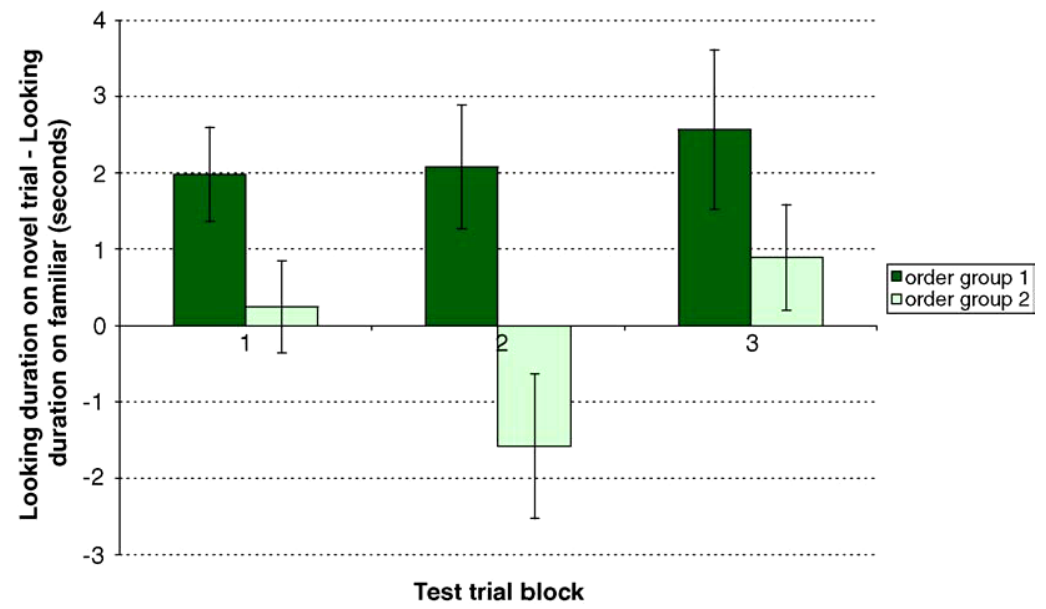

Fig. 3. Novelty preference (difference scores) across blocks 1-3, and between order groups.

The infants demonstrated a novelty preference within these test trial blocks on 37 of the 54 trials. According to a binomial test this proportion of novelty preference is significantly greater than would have been expected by chance $(\mathrm{p}<0.01)$. This coarse analysis corroborates the novelty preference found in the first trial block.

Next, we undertook a full statistical analysis of the mean difference between infants' looking at the novel and familiar OCL configurations across order groups and all test trial blocks. A preliminary ANOVA revealed no significant effects or interactions of OCL comparison, novel OCL, or the starting orientation of the object. The data were thus collapsed across these variables for analysis. A mixed design ANOVA of one within-subjects factor (test trial block (1-3)), and one between-subjects factor (order group (1 or 2)) was carried out on the infants' mean preference scores (looking on novel trial - looking on familiar trial) (see Fig. 3). This analysis uncovered a main effect of order group $(\mathrm{F}(1,16)=7.2, \mathrm{p}<0.017)$. An effect of test block did not reach significance $(\mathrm{F}(2,32)=2.8, \mathrm{p}=0.076)$. There was no significant interaction between order group and test trial block $(\mathrm{F}(2,32)=1.6, \mathrm{n} . \mathrm{s})$.

Closer inspection of the data, however, indicates a heterogeneity of variance between subgroups. The more extreme looking preferences (to novel and familiar stimuli) demonstrate higher variance between subjects. This heterogeneity is observable in Fig. 3, where the more extreme preference means show greater variance. Due to this violation of parametric assumptions, and particularly the borderline nature of the main effect of block we decided to conduct a second analysis of the main effects using non-parametric tests.

A Mann-Whitney test of the effect of order group on the difference score across all test trial blocks confirmed the main order effect shown in the ANOVA $(U=15, Z=2.3, p<0.025)$. A Friedman test for difference in preference scores between test trial blocks (collapsed across both order groups) demonstrated a significant effect of test trial block $(\mathrm{xf}=10.1, \mathrm{~N}=18, \mathrm{p}=0.006)$. Post hoc Wilcoxon signed-ranks tests showed that this effect was largely due to the difference in preference between blocks 2 and $3(Z=2.8, N=18, g=0.005)$. The differences between preference scores in test blocks 1 and $2(Z=1.2, N=18, n . s$.$) , and 1$ and $3(Z=0.9, N=18$, n.s. $)$ were not significant.

One-sample t-tests of the novelty preference within each block, showed that, across both order groups, the infants demonstrated significant novelty preferences in test trial blocks $1(\mathrm{t}(17)=2.4$, $\mathrm{p}=0.03)$ and $3(\mathrm{t}(17)=2.7, \mathrm{p}=0.02)$, but no preference in block $2(\mathrm{t}(17)=0.3, \mathrm{n} . \mathrm{s})$. The significant novelty preferences shown here indicate that 4-month-olds are able to discriminate between objects on the basis of their constant spatial configuration, despite changes in object orientation within the picture plane. Furthermore the modulation of these novelty effects by order indicate that the infants' patterns of looking were sensitive to the temporal relationship between 
novel and familiar test stimuli (Scöhner \& Thelen, in press). Both findings indicate that the infants were able to form a basic object-centred spatial representation.

\section{Discussion}

Following familiarisation to a single object-centred location presented in six different orientations of the object, the infants tested here demonstrated a preference for a novel object-centred spatial configuration over the a familiar one, despite both configurations being presented in novel orientations with respect to the infants' egocentric axes. This result indicates that 4-month-olds are able to notice changes in feature location relative to an object, independently of egocentric and allocentric frames of reference. This novelty preference was significant within the first test trial comparison block, and over all three blocks of test comparisons. Between test trial blocks the infants' preference for novel and familiar were modulated by the order in which the novel and familiar OCLs were presented, indicating that the infants were also sensitive to the temporal relationship between novel and familiar test stimuli. We can conclude that, under these conditions, 4-month-olds can discriminate and remember object-centred spatial coordinates.

We offer two explanations of the effect of order on the novelty preference scores. Firstly, the latency of novel test trials, in relation to familiar in order group 2 means that a novelty preference will be working against any effects of fatigue that accumulate towards the end of the test phase. Secondly, order of presentation is frequently found to have an effect on novelty preferences (Baillargeon, 1987; Baillargeon, Spelke, \& Wasserman, 1985; Johnson et al., 2003;

Rivera, Wakeley, \& Langer, 1999), in which 'novel first' groups show greater novelty preferences than familiar first groups (as was found here). Sch"oner and Thelen (in press) have presented a 'dynamic field theory' of infant habituation in which such order effects are explained by the competition between the inhibitory action of habituation and activation due to the novelty of the test phase itself. Once the test phase had begun in the current experiment, the object no longer changed orientation from trial to trial. It is possible that the novelty activation of the test phase encountered before the first novel stimulus in order group 2 produced enough activation of attention to overrun the effect of habituation to the familiar stimulus. In support of this interpretation, we note that the infants showed their largest overall novelty preference in test trial block 3, where the activation produced by the onset of the test trial phase would be predicted to have the weakest effect.

The ability of 4-month-old infants to represent object-centred spatial relations is informative in several ways. Firstly, our results have implications concerning young infants' ability to use external spatial frames of reference. Kaufman and Needham (1999) showed that 6.5-month-olds are able to use allocentric (or environmental) spatial frames of reference, independently of variation relative to the egocentric field. Our results extend the findings of Kaufman and Needham (1999) by showing that 4-month-olds also have the ability to form spatial representations that are independent of both egocentric and environmental spatial frames of reference. This kind of spatial representation is an essential component of object-centred spatial representation, as objects vary not only with respect to the body but also relative to the environmental framework.

With regard to adult models of visual object recognition our findings suggest that view-invariant representations of objects are available early in development. The infants we tested were able to generalise a familiar object-centred spatial configuration to a completely new stationary orientation of the object. The particular spatial discrimination that the infants used to make this generalization required a simple one-dimensional reference with respect to the object-centred spatial framework. This complexity of representation is ascribed to adults in both viewdependent and view-independent theories of object recognition (Biederman, 1987; Tarr \& Pinker, 1990). Beyond this level of representational complexity, view-dependent theories suggest that objects are recognised by mentally rotation to match against stored egocentric spatial representations of the object. This early ability to represent object-centred spatial frameworks is at odds with Piaget's constructionist account of the development of spatial representation. Piaget proposed that infants' spatial representations were initially restricted to egocentric coordinates, 
with more independent spatial reference arising from infants' active exploration of their environment. At 4 months, infants have a fairly limited repertoire of object-directed manual actions (Hofsten \& Fazel- Zandy, 1984; Hofsten \& R" onnqvist, 1988), and thus it seems unlikely this early ability is developmentally dependent upon construction through manual schemas. In line with the conclusion suggested by a great deal of infancy research over the last 20 years (cf. Baillargeon, 2002; Harris \& Butterworth, 2002; Slater, 1995), active manual exploration does not appear to be a vital prerequisite for learning about objects.

\section{Acknowledgements}

This research was supported by a university scholarship from the University of Oxford, and a postdoctoral research fellowship from the ESRC (T026271357), both awarded to A.B. Additional support was provided by ESRC (UK) grant Roo023911 and European commission RTN grant HPRN-CT-2000-00065. The authors would like to thank the parents and children who participated; Brian Rogers for helpful comments on an earlier draft of this manuscript, and Catherine Crane for assistance in scoring the data.

\section{References}

Acredolo, L. P. (1978). Development of spatial orientation in infancy. Developmental Psychology, 14, 224234 .

Baillargeon, R. (1987). Object permanence in 3.5- and 4.5-month-old infants. Developmental Psychology, $23,655-664$.

Baillargeon, R. (2002). The acquisition of physical knowledge in infancy: A summary in eight lessons. In U. Goswami (Ed.), Blackwell handbook of childhood cognitive development. Oxford, UK: Blackwell Publishing.

Baillargeon, R., Spelke, E., \& Wasserman, S. (1985). Object permanence in 5-month-old infants. Cognition, 20, 191-208. Bertenthal, B. I., Campos, J. J., \& Barrett, K. C. (1984). Self-produced locomotion: An organiser of emotional, cognitive and social development in infancy. In R. Emde \& R. Harnon (Eds.), Continuities and discontinuities in development. New York: Plenum.

Biederman, I. (1987). Recognition-by-components: A theory of human image understanding. Psychological Review, 94, 115-147. Bomba, P. C., \& Siqueland, E. R. (1983). The nature and structure of infant form categories. Journal of Experimental Child Psychology, 35, 294- 328.

Bremner, J. G. (1978). Egocentric versus allocentric coding in nine-month-old infants: Factors influencing the choice of code. Developmental Psychology, 14, 346-355.

Bremner,A. J.,\&Bryant, P. E. (2001). The effect of spatial cues on infants' responses in theABtask, with and without a hidden object. Developmental Science, 4, 408-415.

Bremner, J. G., \& Bryant, P. E. (1977). Place versus response as the basis of spatial errors made by young infants. Journal of Experimental Child Psychology, 23, 162-171.

Butterworth, G., Jarrett, N., \& Hicks, L. (1982). Spatio-temporal identity in infancy: Perceptual competence or cognitive deficit? Developmental Psychology, 18, 435-449.

Gilmore, R. O., \& Johnson, M. H. (1997a). Egocentric action in early infancy: Spatial frames of reference for saccades. Psychological Science, 8, 224-230.

Gilmore, R. O., \& Johnson, M. H. (1997b). Body-centered representations for visually guided action emerge during early infancy. Cognition, 65, 1-9.

Harris, M., \& Butterworth, G. (2002). Developmental psychology: A student's handbook. Hove: Psychology Press, Taylor and Francis.

Hermer, L., \& Spelke, E. (1994). A geometric process for spatial reorientation in young children. Nature, 370, 57-59. 
Hespos, S. J., \& Rochat, P. (1997). Dynamic mental representation in infancy. Cognition, 64, 153-188.

von Hofsten, C.,\&Fazel-Zandy, S. (1984). Development of visually guided hand orientation in reaching. Journal of Experimental Child Psychology, 38, 208-219.

von Hofsten, C., \& R“onnqvist, L. (1988). Preparation for grasping an object: A developmental study. Journal of Experimental Psychology: Human Perception and Performance, 14, 610-621.

Hummel, J. E. (2000). Where view-based theories break down: The role of structure in human shape perception. In E. Dietrich \& A. B. Markman (Eds.), Cogntive dynamics: Conceptual change in humans and machines. Mahwah, NJ: Erlbaum.

Johnson, S. P., Bremner, J. G., Slater, A. M., Mason, U. C., Foster, K., \& Cheshire, A. (2003). Infants' perception of object trajectories. Child Development, 74, 94-108.

Kaufman, J., \& Needham, A. (1999). Objective spatial coding by 6.5-month-old infants in a visual dishabituation task. Developmental Science, 2, 432-441.

Marr, D., \& Nishihara, H. K. (1978). Representation and recognition of the spatial organisation of threedimensional structure. Proceedings of the Royal Society of London, Series B (Biological Sciences), 200, 269-294.

Mozer, M. C. (2002). Frames of reference in unilateral neglect and visual perception: A computational perspective. Psychological Review, 109, 156-185.

Newcombe, N. S. (2003). The nativist-empiricist controversy in the context of recent research on spatial and quantitative development. Psychological Science, 13, 337-341.

Newcombe, N. S., \& Huttenlocher, J. (2000). Making Space: The development of spatial representation and reasoning. Cambridge, MA: MIT Press.

Piaget, J. (1952). The origins of intelligence in the child. London, UK: Routledge \& Kegan-Paul (Originally published in French in 1936).

Piaget, J. (1954). The construction of reality in the child. London, UK: Routledge \& Kegan-Paul (Originally published in French in 1937).

Quinn, P. C., Slater, A. M., Brown, E., \& Hayes, R. A. (2001). Developmental change in form categorisation in early infancy. British Journal of Developmental Psychology, 19, 207-218.

Quinn, P. C., Eimas, P. D., \& Tarr, M. J. (2002). Perceptual categorisation of cat and dog silhouettes by 3- to 4-month-old infants. Journal of Experimental Child Psychology, 79, 78-94.

Rochat, P.,\&Hespos, S. J. (1996). Tracking and anticipation of invisible spatial transformations by 4- to 8month-old infants. Cognitive Development, 11, 3-17.

Rivera, S. M.,Wakeley, A., \& Langer, J. (1999). The drawbridge phenomenon: Representational reasoning or perceptual preference? Developmental Psychology, 35, 427-435.

Sch“oner, G., \& Thelen, E. (in press). Using dynamic field theory to rethink infant habituation. Psychological Review. Slater, A. M. (1995). Visual perception and memory at birth. In C. Rovee-Collier \& L. P. Lipsitt (Eds.), Advances in infancy research: Vol. 9. Norwood NJ: Ablex.

Slater, A. M., Mattock, A., \& Brown, E. (1990). Size constancy at birth: Newborn infants' responses to retinal and real size. Journal of Experimental Child Psychology, 49, 314-322.

Slater,A. M., Mattock, A., Brown, E.,\&Bremner, J.G. (1991). Form perception at birth revisited: Cohen andYounger (1984). Journal of Experimental Child Psychology, 51, 395-405.

Slater, A. M., \& Morison, V. (1985). Shape constancy and slant perception at birth. Perception, 14, 337-344.

Tarr, M. J. (1999). News on views: Pandemonium revisited. Nature Neuroscience, 2, 932-935. 
Tarr, M. J., \& Bulthoff, H. H. (1998). Image-based object recognition in man, monkey, and machine. In M. J. Tarr \& H. H. Bulthoff (Eds.), Object recognition in man, monkey, and machine. Cambridge, MA: MIT Press.

Tarr, M. J., \& Pinker, S. (1990). When does human object recognition use a viewer-centred reference frame? Psychological Science, 1, 253-256.

Tarr,M. J.,Williams, P., Hayward,W. G., \& Gautier, I. (1998). Three-dimensional object recognition is viewpoint dependent. Nature Neuroscience, 1, 275-277.

Tipper, S. P., \& Behrmann, M. (1996). Object-centred not scene-based visual neglect. Journal of Experimental Psychology: Human Perception and Performance, 22, 1261-1278.

Vecera, S. P., Behrmann, M., \& Filapek, J. C. (2001). Attending to the parts of a single object: Part-based selection limitations. Perception and Psychophysics, 63, 308-321. 